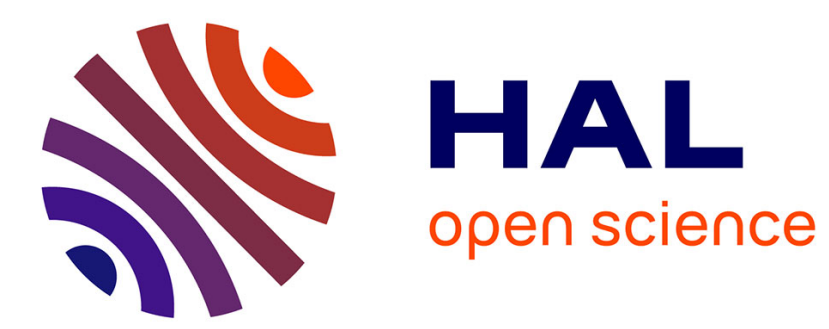

\title{
la langue de l'exilé
}

Michèle Benhaim

\section{To cite this version:}

Michèle Benhaim. la langue de l'exilé . Cliniques méditerranéennes, 2001, 10.3917/cm.064.0097 . hal-01429359

\section{HAL Id: hal-01429359 https://hal-amu.archives-ouvertes.fr/hal-01429359}

Submitted on 7 Jan 2017

HAL is a multi-disciplinary open access archive for the deposit and dissemination of scientific research documents, whether they are published or not. The documents may come from teaching and research institutions in France or abroad, or from public or private research centers.
L'archive ouverte pluridisciplinaire HAL, est destinée au dépôt et à la diffusion de documents scientifiques de niveau recherche, publiés ou non, émanant des établissements d'enseignement et de recherche français ou étrangers, des laboratoires publics ou privés. 


\section{LA LANGUE DE L'EXILÉ}

Michèle Benhaïm

\section{ERES | «Cliniques méditerranéennes »}

2001/2 nº $64 \mid$ pages 97 à 106

ISSN 0762-7491

ISBN 2-86586-890-7

Article disponible en ligne à l'adresse :

http://www.cairn.info/revue-cliniques-mediterraneennes-2001-2-page-97.htm

\section{Pour citer cet article :}

Michèle Benhaïm, « La langue de l'exilé 》, Cliniques méditerranéennes 2001/2 (nº 64), p. 97-106.

DOI $10.3917 / \mathrm{cm} .064 .0097$

Distribution électronique Cairn.info pour ERES.

(C) ERES. Tous droits réservés pour tous pays.

La reproduction ou représentation de cet article, notamment par photocopie, n'est autorisée que dans les limites des conditions générales d'utilisation du site ou, le cas échéant, des conditions générales de la licence souscrite par votre établissement. Toute autre reproduction ou représentation, en tout ou partie, sous quelque forme et de quelque manière que ce soit, est interdite sauf accord préalable et écrit de l'éditeur, en dehors des cas prévus par la législation en vigueur en France. Il est précisé que son stockage dans une base de données est également interdit. 


\section{Michèle Benhaïm}

\section{La langue de l'exilé}

Ce texte est dédié à Jacques Hassoun

\section{INTRODUCTION}

Qu'est-ce qui se transmet de la migration des parents aux enfants et qui semble participer de processus de désubjectivation, de désidentification de l'adolescent?

Pour tenter d'en saisir quelques éléments, il nous faut commencer par différencier migration et exil et se demander de l'exil de qui l'on parle.

Si migrer consiste à quitter un lieu pour un autre, parler de l'exil, c'est témoigner d'une souffrance, celle d'un arrachement, d'une absence.

Nous pouvons dans certains cas avancer l'hypothèse suivante : les exilés, aujourd'hui, sont les enfants d'immigrés. En effet, l'« exil » de l'adolescent, fils de migrants, peut trouver son expression dans son incapacité à identifier et à nommer les raisons de son errance.

Nous évoquerons deux exemples cliniques : le premier, Shéhérazade, montrera comment il est possible de ne pas s'inscrire fatalement à cette place, c'est-à-dire comment l'immigration des parents n'exile pas nécessairement les enfants, si ces derniers ne s'inscrivent pas dans le deuil et la problématique parentaux, et, qu'à l'inverse, ils " choisissent » de s'enrichir de l'addition entre une culture d'origine et une culture d'accueil, de ne pas s'exiler d'eux-mêmes et de se constituer de leurs compléments.

Mais souvent, on ne peut désintriquer le malaise filial du malaise social et, si l'on interroge les mots qui assignent ces adolescents, "seconde génération ", sous entendu « d'immigrés », on peut, cette fois sur le versant de notre hypo- 
thèse, les articuler à la problématique du jeune qui se débat entre le statut conjoncturel de ses parents, la migration, et ce qui lui en est transmis sous les traits d'un exil douloureux, générant ce mal-être identitaire qui vient signifier, finalement, comment la filiation aux racines n'a pu être préservée.

Le second exemple étayera par contre l'hypothèse en montrant comment Sofia peut articuler sa problématique adolescente organisée par un effondrement parental (dont on sait les effets dramatiques que ce dernier peut engendrer durant ce temps de passage pour le sujet) sur ce que peut exacerber le fait que cet effondrement prenne place dans un contexte d'immigration et d'exil parental, télescopant nécessairement la question universelle autour de l'origine, que l'adolescent ne se lasse pas de poser.

Le symptôme, expression de la rupture mais aussi tentative de suture, venant souvent " résoudre » momentanément la question, révèle que l'adolescent, plus qu'entre deux cultures, se trouve pris entre deux impossibles : d'une part ne pas «trahir », non pas son origine mais une illusion parentale à laquelle plus personne ne croit et qui peut se traduire par le déclin d'un père que le " choc à l'arrivée » a rendu « silencieux » et la dépression d'une mère endeuillée, captive du paradoxe de ne pouvoir dénouer les éléments de ce deuil ; d'autre part s'intégrer, c'est-à-dire se fondre, voire se confondre à un autre, alors même que le côtoiement de cet autre semble exacerber le rapport forcement ambivalent à sa propre culture, raviver les failles subjectives et renvoyer chacun à ses propres manques, aux zones d'ombres de sa singularité.

\section{SHÉHÉRAZADE}

Ce que Shéhérazade décrit sous le terme d' « incompatibilité » pour évoquer quelque chose de l'impossible rencontre, lorsque l'un, vécu comme étranger, n'est pas reconnu comme porteur d'altérité, lorsqu'une frontière, une limite est tracée entre elle et l'autre au-delà de laquelle il la situe comme impensable, c'est-à-dire hors-lieu, hors-loi.

Shéhérazade qui énonce d'emblée : « Mon père est émigré, ma mère est exilée » illustre par ses propos comment elle-même, au lieu de se résoudre au compromis symptomatique, invente son désir, plus que sa culture, et s'attache à transformer, dans des ajustements quotidiens, la déchirure maternelle en ouverture et en liens préservés avec l'origine d'une part, avec le lieu de naissance, d'autre part.

«Ma mère a peur que je me sente française. Moi je sais d'où je viens. Elle "rentre" en Algérie. Moi, je "rentre" là-bas mais je "rentre" aussi en France. Je me sens les deux : fille d'immigrés en Algérie et fille d'immigrés en France, algérienne et française et non pas l'une ou l'autre ou encore "ni l'une ni l'autre" ", c'est-à-dire figée dans le nulle part. 
Elle se sauve de se sentir «fille de » et rompt par là la coupure de l'exil dans sa dimension asubjectivante, en s'épanouissant autant intellectuellement que relationnellement.

Shéhérazade illustre de façon heureuse comment là où il y a trop souvent de la perte, pour elle il y a du plus de créativité, au sens de Winnicott, comment là où tout aurait pu signer de la désaffiliation, Shéhérazade chemine à la rencontre de son désir singulier qui vise à bâtir des passages et des complétudes, à engager le dialogue sans trahir, c'est-à-dire sans adopter le tout de l'autre.

Ce processus repose sur la façon dont elle fait communiquer en elle la langue arabe et la langue française, reliant dans ce balancement l'avant et l'actuel, le familier et l'étranger, le dedans et le dehors, habitant un espace « dedans le dehors » parental, le proche et le lointain et enfin le père à la mère comme dans une recomposition triangulaire relative aux deux pays, ce qui lui permet de faire émerger pour elle-même une identité propre qui en passe, comme tout processus subjectivant, par la résurgence de conflits ambivalents.

Nous verrons comment le regard de l'autre peut reconstruire des frontières dans la tête du sujet, rendre le sujet étranger au point de le devenir à lui-même. Également comment le travail d'analyse avec ces adolescents peut aider à la symbolisation, à la pacification des espaces, des temps, des histoires singulières en les désintriquant des histoires parentales, autrement dit en séparant l'enfant du désir de sa mère.

" Je me sens algérienne parce que ma famille est algérienne mais si je tombe amoureuse d'un garçon français, je n'y peux rien, je crois en l'amour ", sont les propos tenus par Shéhérazade qui laissent percevoir la façon dont elle transforme la souffrance de l'exil en une possibilité d'accueillir de nouveaux liens.

Alors qu'est-ce qui, d'une migration parentale non élaborée en son temps, laisse aujourd'hui l'adolescent désemparé, c'est-à-dire sans défenses, ou pire, le précipite dans une angoisse massive et catastrophique, pouvant le mener à quitter, pour le coup tous les lieux?

SOFIA

Je pense ici à Sofia, rescapée d'une expérience extrême de non-appartenance au monde, en l'occurrence d'une chute ayant consisté à se jeter du haut de la «Bonne-Mère ». Comme si, pour elle, avait été paralysé le processus métaphorique et qu'avaient ainsi manqué les mots qui parlent à la fois le maintien de la relation à la mère et à la fois la séparation d'avec la mère. Comme si l'on ne pouvait introjecter un monde d'où l'on est, en réalité, absent. 
Peut-il être question de filiation hors-symbolique, c'est-à-dire hors-séparation d'avec la mère?

Le deuil maternel non élaboré avait pris des allures de désinvestissement du présent qui s'exprimait dans une angoisse d'abandon, elle-même sous-jacente à un comportement de rejet brutal vis-à-vis de Sofia. Cette fêlure, cet appel, de n'avoir pas trouvé de lieu de reconnaissance et d'écoute, semble avoir été vécu par la mère comme un insupportable.

Sofia articulera son passage à l'acte au fait qu'elle était devenue l'espace de projection privilégié de l'angoisse maternelle que rien ne parvenait plus à contenir. Quant à son père alors : «Je ne sais pas ce qu'il veut, ni ce qu'il fait, il ne faisait rien $»$.

Une mère endeuillée, un père éclipsé comme sujet dans le social et dans son rapport à ses objets font désastre pour Sofia. Adolescente abandonnée à son vertige, elle entame alors une errance toxicomaniaque qui, en lui permettant d'interrompre les questions : " Où suis-je ? Comment parler avec tout ça ? Comment parler avec ce que mes parents ne veulent pas me dire, ne peuvent pas me transmettre ? ", traduit le passage d'un pays à un autre par un exil qui lui revient et qui se signe d'une mise en non-lieu.

La chute comme le résultat peut-être d'une mémoire impossible, n'offrant à un désir de lieu, nul lieu pour que ce désir prenne corps.

$C^{\prime}$ est à l'adolescence, au temps de la recherche et de la consolidation du sentiment d'identité, c'est-à-dire de ce qui fonde la relation entre un individu et son groupe, au temps de la circulation des désirs dans une économie psychique familiale aux enjeux bouleversés, c'est enfin dans ce temps des deuils adolescents multiples que celui, non élaboré des parents, a confronté Sofia à l'épreuve de l'exil et de la non-reconnaissance dans le réel.

Nous savons que souvent, à l'adolescence des enfants se réactive la dépression parentale dans un heurt entre les deuils pubertaires en voie d'accomplissement et justement les pertes qui n’ont pas pu être élaborées en travail de deuil.

Dans ce passage où l'adolescent s'interroge sur son rapport en tant que sujet à un groupe, y compris au groupe familial, les parents, en le chargeant d'une tâche psychique supplémentaire, le tirent hors du groupe pour l'investir de leur exil.

Autrement dit, voici le sujet adolescent pris dans ce que Winnicott appellerait une " crainte de l'écroulement » dont il précise qu'il répète un écroulement déjà expérimenté, et, mis en demeure de poser une double question au père : celle qui interroge son rapport de soumission au phallus mais aussi celle qui s'étonne ou s'indigne de sa soumission à une logique sociale qui l'exclut. 
Que peut l'adolescent, ainsi captif, s'approprier de sa propre division ? Sofia l'a-t-elle joué dans son corps en expérimentant le trou et le manque dans son parcours toxicomaniaque?

On peut supposer en tout cas que, devenue adolescente affirmant avec violence son désir d'autonomisation dans une dimension extrême ne laissant nulle place à la tradition, elle a redoublé la fracture parentale et placé ses parents face à leur propre ambivalence quant au retour dont ils parlaient souvent mais qu'ils ne réalisaient jamais, dans lequel ils ne se retrouvaient plus au niveau de leur désir, de leur propre histoire actuelle, elle a fait intrusion comme une insulte en eux d'un pays "renié ».

Sofia alors confrontée à la défaillance subjective de sa mère, angoissée et déprimée, et à celle de son père qui, suite à un début de cancer, était en train de perdre son emploi, défaillances que rien n'est venu nommer, ni élaborer, elle en est devenue la dépositaire.

Une adolescente, face à un vide de référence, paye la dette de non-transmission parentale de sa douleur, de sa folie ou de sa mort.

\section{LE CORPS}

Si Sofia a miraculeusement survécu aux trois semaines de coma consécutifs à sa chute, son corps demeure marqué par cet exil radical venu se fracasser sur un " passage adolescent » au cours duquel le sujet pose, entre autres, la question de la transmission d'une génération à l'autre. Rien n'est venu répondre dans les registres imaginaire et symbolique du lien, le seul point d'accueil a relevé du réel d'une chute. «Écroulement » dans le réel en écho à l'écroulement qui le précède, celui de la mère « exilée » dans l'imaginaire.

Tentative de faire échouer radicalement toute possibilité d'altérité, comme la toxicomanie en avait, chez Sofia, tracé l'esquisse, celle d'une violence sans discours, lui évitant de surcroît d'avoir à " choisir » sa langue.

Souvent, comme pour Sofia, le corps est ce qui continue de témoigner de ce travail de deuil non effectué, transmis à travers les générations.

C'est au même temps, celui de l'adolescence, que pour d'autres, l'acte sera posé par les parents, prenant, par exemple, la forme d'un « retour » forcé au pays, de l'adolescent qui parfois ne connaît même pas le pays et serait bien en peine $d^{\prime} y$ faire retour. On le ramène pour ne pas qu'il perpétue la perte, perte des racines, de la langue, des traditions, de la famille, restée làbas, des tombes ancestrales trop éloignées. Mourir dans l'esprit des proches que l'on quitte, est pure perte et douleur, et ouvre dans l'être une béance qui sera tenue pour responsable de la catastrophe imaginaire qui s'ensuit. 
Dans la famille de Sofia, plusieurs éléments comme une constellation de facteurs de peine latents, convergent vers l'hypothèse que le deuil engendré par le processus migratoire parental, lorsqu'il est laissé en suspens sur la scène de l'ailleurs se retrouve intact et à faire lorsque les enfants, devenus adolescents, affirment leur désir de vivre là où ils sont nés et là où ils ont grandi. Ailleurs, se construit la maison sensée accueillir le retour, abritant, dans cette attente, les racines familiales.

Parents en deuil, enfants nés en Tunisie en deuil, enfants nés en France " exilés », chaque clandestin de la vie en perte d'identité, se débat à une étape différente de la perte. Peut-être est-ce dans ce temps que la « douleur de leur cœur devient clairement une dépression » pour citer Freud évoquant son propre exil à la fin de sa vie, et que les parents délèguent alors à la « seconde génération » le soin d'en métaphoriser quelque chose.

Chez Sofia, le premier garçon né en France s'électrocute à l'âge de 17 ans et Sofia, première fille née en France, tente un suicide qui laissait, a priori, peu de chances d'en réchapper, actes dont on pourrait interroger l'accent sacrificiel. Sacrifier l'enfant pour retrouver l'origine supposée perdue à la génération précédente, ou encore, (pour emprunter aux travaux de Martine Broda), " pour consoler l'ange perdu dont je porte le deuil, il faut qu'une enfance agonise ».

Les parents et la génération née en Tunisie ont un rapport avec un objet perdu connu. Les enfants nés en Tunisie sont ceux du dedans, aimés par les parents. Les aînés nés en France s'inscrivent dans une généalogie menacée dans sa continuité par la migration d'une part, par la fragilité parentale qui en découle d'autre part.

Comment la mère de Sofia aurait-elle pu alors produire une métaphore qui aurait pu lier le deuil à la grossesse ? Comment et que transmettre au sein d'une telle insécurité, comment investir l'enfant qui naît quand l'essentiel est demeuré dans cet ailleurs ? La filiation consiste quand même à s'inscrire dans la trace de quelqu'un. Entre le dedans et le dehors semble passer la frontière de la lignée filiale.

\section{L'ESPACE THÉRAPEUTIQUE}

L'espace de travail thérapeutique vise à aider Sofia à construire son espace identitaire sans sacrifier son individualisation acquise, ni trahir son origine passée. Pour Sofia pour l'instant c'est l'un ou l'autre. Elle a « choisi » : "Je hais les arabes », me dit-elle souvent lorsqu'elle veut résumer qu'elle hait presque tous les membres de sa famille.

À travers une élaboration de la violence de cet écart, comme chacun elle chemine cependant vers l'inscription de son désir propre dans une filiation, 
c'est-à-dire un groupe familial non haï et dans une affiliation à un groupe social.

L'espace thérapeutique lui offre dans un premier temps la possibilité de s'éprouver à l'altérité comme une expérience non mortelle, comme une alternative, un lieu d'élaboration et de réalisation des possibles, une continuité dans la rupture. Plutôt que la haine, une redéfinition de ce qui se transmet des uns aux autres.

D'abord figée dans l'ailleurs au détour de la drogue, puis s'immobilisant dans le présent au travers du passage à l'acte, Sofia témoigne peut-être que ce qui fut atteint a concerné la question de la filiation actuelle mais aussi celle de la filiation à venir.

Les séquelles corporelles témoignent quant à elles de la difficulté à définir la nature de la douleur de l'exil, douleur à la limite du mental et du physique, rendant peut-être si difficile la métaphore du départ.

Le corps de la mère traversé de plaintes adressées à l'enfant, le corps du père dévasté par la maladie grave, le corps du frère figé à jamais sous ses yeux de petite fille, le corps de Sofia enfin, brisé sur l'écueil des deuils d'autant plus pathologiques que les sujets n'ont pu les reconnaître, les sentir, les exprimer, c'est-à-dire produire l'immense effort psychique nécessaire à l'acceptation de la perte et aux retrouvailles du lien affectif avec la réalité.

Sofia a fait de son corps exilé souffrant dans un gel psychique le lieu même de l'être étranger. Prise de surcroît dans des processus d'exclusion sociale, comment aurait-elle pu s'approprier ce corps, comme lieu d'une parole, à la fois en écho et en rupture avec la voix de l'origine?

Adolescente entre deux, certes, mais peut-être pas tant entre deux cultures qu'entre deux abandons : le regard maternel tourné vers un paradis dont il est difficile de symboliser la perte, percutant le regard que l'autre social renvoie et qui peut être chargé de menaces chaotiques n'offrant aucune issue au drame de l'exil.

Le sentiment de dignité et d'estime de soi se tisse dans le regard d'autrui tout comme l'unité du corps prenait sens dans le regard maternel.

Parfois, rien ne vient ouvrir du dehors ce que le dedans n'a pu transmettre que comme une clôture. C'est ce en quoi le regard raciste participe de l'exil de sens pour l'adolescent. Il relève d'une double désaffiliation, celle des parents, communauté d'origine dont on a honte, celle de la communauté $\mathrm{d}^{\prime}$ accueil dont on est exclu. Le social aurait pourtant pu, comme pour Shéhérazade étayer et relayer ce que cristallisent les douleurs et les ruptures familiales. C'est à cet endroit que le malaise filial se noue au malaise social comme je l'avançais tout à l'heure.

Pour Sofia, comme pour beaucoup d'autres, la révolte exacerbée contre la famille recouvrait sans doute son malaise identitaire s'originant dans la 
situation d'exil, non-lieu déserté par la référence symbolique de la culture d'origine, en l'occurrence le lien transgénérationnel demeuré vide des références de la culture d'accueil.

L'arrachement ne vaut que s'il est une avancée vers l'autre sans pour autant signer un renoncement à soi-même. À défaut, les jeunes n'en finissent pas de s' « arracher » d'un réel social où peu de choses étayent la peine et l'espoir, les contiennent ou aident à les transformer, $s^{\prime}$ « arracher » d'un regard qui atteint le sentiment de continuité et d'être toujours soi-même.

Quand l'objet extérieur devient persécuteur, peuvent surgir la honte et le désir d'obliger le monde à ne pas nous regarder, ou bien la haine d'un monde sans contenance.

Quand l'enfant pleure et que la mère n'accourt pas, il peut croire qu'il n'existe pas pour la mère. Les « sans-papiers » à Marseille ont fait un film pour, précisent-ils, «faire la preuve de leur existence ».

Le sujet peut souffrir d'être confronté à un point déshumanisant qui condense la souffrance individuelle et le bannissement collectif. Il évoque alors comme une crainte d'être anéanti dans l'impossibilité de se repérer et de repérer autrui. Si le sentiment d'identité se développe en se fondant sur le lien avec les autres, un lien social sinistré engendre des risques de dépersonnalisation.

Un travail psychique relatif à une inscription filiale n'aurait pu exister pour Sofia qu'au sein d'une appartenance sociale, d'un étayage culturel et non pas dans un processus d'une part de désidentification à partir du rejet d'une mère devenue « insuffisamment bonne » et, d'autre part de disqualification de l'ordre paternel engendré par la perte d'emploi relative à la maladie invalidante. Pour Sofia, cette double délégitimation s'est muée en un refus radical de l'origine et de tous les signes qui la trahissent.

Qu'en est-il du sujet lorsqu'il se trouve réduit par un autre à ne plus être représenté que par un système de signes de désignation péjorative ? Sofia rejette tout en bloc, dans un même mouvement, y compris elle-même. Comme Wolfson elle ne peut plus parler la langue de la mère, ni même l'entendre, surtout énoncée par la mère. Dans une tentative désespérée de survivre à la haine maternelle et à la haine du regard de l'autre, elle s'approprie ce qu'elle croit être les signifiants privilégiés de l'autre (drogue, alcool, fumer ostensiblement devant le père.)

Le cercle se referme quand Sofia, ainsi affublée, ne peut plus être reconnue par ses parents que comme une fille illégitime.

Depuis son saut, Sofia a du mal à parler, ne trouve plus ses mots comme si la menace de mort avait entraîné une démétaphorisation du langage. Parfois, dans la psychothérapie, ne peuvent surgir que des mots en arabe, isolés, désignant des bouts de réel. L'impossibilité de métaphoriser les enjeux 
de la migration semble faire défaillir la langue. Parfois elle dit qu'elle voudrait changer de nom, désaveu de la filiation aussitôt escorté de sentiments coupables.

Le symptôme adolescent pose une exigence de justice généalogique. Aussi le rapport du sujet à sa généalogie se complique-t-il si le social, sensé être civilisateur, ne permet pas d'identifier en quoi son être dans la filiation participe du symbolique.

Un conflit public/subjectif émerge dans l'intime du sujet. Parfois on a le sentiment que les blessures sont telles que l'on ne parviendra plus à relancer liens et projets dans un élan vital. C'est ce temps où l'adolescent se fige, comme le parent, dans un indicible. Seule alors l'initiative de la rencontre laissera espérer une remise en mouvement de la demande.

Le lieu psychothérapeutique permettra alors de reprendre autrement et peut-être plus profondément substances et racines. On peut assister à un réenracinement, un mouvement de passage chez le jeune qui se met pour le coup à transmettre. C'est la place que commence timidement à occuper Sofia à qui les sœurs aînées et les parents demandent de l'aide.

Restituer les racines aux parents et construire de l'histoire aux enfants fait sortir de l'impossible sur un autre mode que celui de la culpabilité, de la honte ou de la haine. Le travail psychique consiste en un mode d'appropriation des éléments de l'origine et des éléments de l'actuel, éléments qui convergent vers une identité possible pour le sujet dans son rapport au monde.

Transformer le paradis perdu en passé offre la possibilité de n'avoir à renoncer ni à la culture nouvelle, ni à la culture d'origine, interrompt de la sorte les ruptures de transmissions, permet de s'approprier l'acte migratoire parental en en élaborant l'héritage plutôt que de tenter d'en abolir la réalité.

Sofia s'agrippe à moi comme à une surface d'identification projective et tente ainsi de reconstruire sa capacité d'expérience culturelle. La rencontre vient signifier que tout n'est pas mort, ni tué. L'expérience de la parole ici et maintenant est nécessaire à ce que la séparation ait lieu dans un mouvement de vie et non dans un déchaînement mortifère de haine. Pour que les retrouvailles avec la mère aient lieu, nous savons qu'il faut d'abord s'en être séparé symboliquement, c'est-à-dire en s'inscrivant dans une filiation.

\section{CONCLUSION}

Le risque pour le thérapeute est de tomber dans l'écueil de l'exil comme écran au sujet et à ses événements psychiques singuliers. Bien que l'on s'attache à construire un espace où l'on continuera d'entendre ce qui insiste de la dimension subjective, la clinique témoigne aujourd'hui que l'exil en tant 
que tel peut mener le sujet à faire des choix inédits et à occuper des positions subjectives inédites, où justement s'entremêlent les question de filiation et d'affiliation.

\section{Résumé}

Ce qui se transmet de la migration des parents aux enfants peut faire s' « exiler » un adolescent pris entre du deuil maternel inélaborable et un impossible social. Exil qui s'actualise soit dans une conduite symptomatique, soit dans un processus de création, quand l'adolescent a pu transformer la déchirure en ouverture.

À partir d'une pratique clinique avec des jeunes dont les parents ont migré, l'auteur essaie d'apporter quelques éléments permettant de saisir ce qui se " passe ».

\section{Mots clés}

Migration, exil, malaise filial, malaise social, deuil maternel, adolescence, mémoire, corps, espace thérapeutique.

\section{THE LANGUAGE OF THE EXILE}

\section{Summary}

What is handed down to their children by parents' migration can lead an adolescent to « exile » from himself, because of being taken between an inelaborated maternal's mourning and something socialy impossible. Exile which will update, either in a symptomatic behaviour, or in a process of creation when the teenager will be able to transform the heartbreack into an openmindedness.

From a clinical practice with young people whose parents had migrated, we will try to bring some elements allowing to seize what's " passing ».

\section{Key words}

Migration, exile, filial uneasiness, social uneasiness, maternal's mourning, adolescence, memory, body, therapeutic space. 